\title{
Modular Identities and Explicit Evaluations of a Continued Fraction of Ramanujan
}

\section{Nipen Saikia}

Department of Mathematics, Rajiv Gandhi University, Rono Hills, Doimukh-791112, Arunachal Pradesh, India

Correspondence should be addressed to Nipen Saikia, nipennak@yahoo.com

Received 23 March 2012; Accepted 11 June 2012

Academic Editor: Stefaan Caenepeel

Copyright (C) 2012 Nipen Saikia. This is an open access article distributed under the Creative Commons Attribution License, which permits unrestricted use, distribution, and reproduction in any medium, provided the original work is properly cited.

We study a new continued fraction of Ramanujan. We prove its modular identities and give some explicit evaluations.

\section{Introduction}

Throughout the paper, we assume $|q|<1$. As usual, for positive integers $n$ and any complex number $a$, we write

$$
(a)_{n}:=(a ; q)_{n}:=\prod_{j=0}^{n-1}\left(1-a q^{j}\right), \quad(a)_{\infty}:=(a ; q)_{\infty}:=\prod_{n=0}^{\infty}\left(1-a q^{n}\right) .
$$

Ramanujan's general theta-function $f(a, b)$ is defined by

$$
f(a, b):=\sum_{k=-\infty}^{\infty} a^{k(k+1) / 2} b^{k(k-1) / 2}
$$


where $|a b|<1$. After Ramanujan, we define

$$
\begin{gathered}
\phi(q):=f(q, q)=1+2 \sum_{k=1}^{\infty} q^{k^{2}}=\frac{\left(-q ; q^{2}\right)_{\infty}\left(q^{2} ; q^{2}\right)_{\infty}}{\left(q ; q^{2}\right)_{\infty}\left(-q^{2} ; q^{2}\right)_{\infty}} \\
\psi(q):=f\left(q, q^{3}\right)=\sum_{k=0}^{\infty} q^{k(k+1) / 2}=\frac{\left(q^{2} ; q^{2}\right)_{\infty}}{\left(q ; q^{2}\right)_{\infty}} \\
f(-q):=f\left(-q,-q^{2}\right)=\sum_{k=0}^{\infty}(-1)^{k} q^{k(3 k-1) / 2}+\sum_{k=1}^{\infty}(-1)^{k} q^{k(3 k+1) / 2}=(q ; q)_{\infty} \\
x(q)=\frac{f(q)}{f\left(-q^{2}\right)}=\left(-q ; q^{2}\right)_{\infty} .
\end{gathered}
$$

Ramanujan recorded many $q$-continued fractions and some of their explicit values in his second notebook [1] and in his lost notebook [2]. The following beautiful continued fraction identity was recorded by Ramanujan in his second notebook and can be found in [3, p. 11, Entry 11]:

$$
\frac{(-a)_{\infty}(b)_{\infty}-(a)_{\infty}(-b)_{\infty}}{(-a)_{\infty}(b)_{\infty}+(a)_{\infty}(-b)_{\infty}}=\frac{a-b}{1-q}+\frac{(a-b q)(a q-b)}{1-q^{3}}+\frac{q\left(a-b q^{2}\right)\left(a q^{2}-b\right)}{1-q^{5}}+\cdots
$$

where either $q, a$, and $b$ are complex numbers with $|q|<1$, or $q$, $a$, and $b$ are complex numbers with $a=b q^{m}$ for some integer $m$. Several elegant $q$-continued fractions have representations as $q$-products and some of them can be expressed in terms of Ramanujan's theta-functions. An account of this can be found in in Chapter 32 of Berndt's book [4] (also see [5]). The most famous one, of course, is the Rogers-Ramanujan continued fraction $R(q)$ defined by

$$
R(q):=\frac{q^{1 / 5}}{1}+\frac{q}{1}+\frac{q^{2}}{1}+\frac{q^{3}}{1}+\cdots
$$

The continued fraction $R(q)$ has a very beautiful and extensive theory almost all of which was developed by Ramanujan. In particular, his lost notebook [2] contains several results on the Rogers-Ramanujan continued fraction. We refer to the paper by Berndt et al. [6], Kang $[7,8]$ for proofs of many of these theorems.

In this paper, we examine another continued fraction $T(q)$ of Ramanujan arising from (1.7) and is defined by

$$
T(q):=\frac{q}{1-q^{2}}+\frac{q^{4}}{1-q^{6}}+\frac{q^{8}}{1-q^{10}}+\cdots
$$

Note that, replacing $q$ by $q^{2}$ and then setting $a=q$ and $b=0$ in (1.7), we obtain (1.9).

In Section 2, we record some preliminary results. Section 3 is devoted to prove some modular identities for the continued fraction $T(q)$. Finally, in Section 4, we give some explicit evaluations of $T(q)$. 
We complete this introduction by defining Ramanujan's modular equation from Berndt's book [3]. The complete elliptic integral of the first kind $K(k)$ is defined by

$$
K(k):=\int_{0}^{\pi / 2} \frac{d \phi}{\sqrt{1-k^{2} \sin ^{2} \phi}}=\frac{\pi}{2}{ }_{2} F_{1}\left(\frac{1}{2}, \frac{1}{2} ; 1 ; k^{2}\right),
$$

where $0<k<1,{ }_{2} F_{1}$ denotes the ordinary or Gaussian hypergeometric function. The number $k$ is called the modulus of $K$, and $k^{\prime}:=\sqrt{1-k^{2}}$ is called the complementary modulus. Let $K, K^{\prime}, L$, and $L^{\prime}$ denote the complete elliptic integrals of the first kind associated with the moduli $k, k^{\prime}, l$, and $l^{\prime}$, respectively. Suppose that the equality

$$
n \frac{K^{\prime}}{K}=\frac{L^{\prime}}{L}
$$

holds for some positive integer $n$. Then, a modular equation of degree $n$ is a relation between the moduli $k$ and $l$ which is implied by (1.11). If we set

$$
q=\exp \left(-\pi \frac{K^{\prime}}{K}\right), \quad q^{\prime}=\exp \left(-\pi \frac{L^{\prime}}{L}\right)
$$

we see that (1.11) is equivalent to the relation $q^{n}=q^{\prime}$. Thus, a modular equation can be viewed as an identity involving theta-functions at the arguments $q$ and $q^{n}$. Ramanujan recorded his modular equations in terms of $\alpha$ and $\beta$, where $\alpha=k^{2}$ and $\beta=l^{2}$. We say that $\beta$ has degree $n$ over $\alpha$. The multiplier $m$ connecting $\alpha$ and $\beta$ is defined by

$$
m=\frac{K}{L}
$$

where $z_{r}=\phi^{2}\left(q^{r}\right)$.

\section{Preliminary Results}

In this section, we record some results that will be used in the subsequent sections.

Lemma 2.1 (see [3, p. 124, Entry 12(i) and (ii)]). One has

$$
f(q)=\sqrt{z}_{1} 2^{-1 / 6}\{\alpha(1-\alpha)\}^{1 / 24} q^{-1 / 24}, \quad f(-q)=\sqrt{z}_{1} 2^{-1 / 6}(1-\alpha)^{1 / 6} \alpha^{1 / 24} q^{-1 / 24} .
$$

Lemma 2.2 (see [3, p. 214, Entry 24(iii)]). If $\beta$ has degree 2 over $\alpha$, then

$$
\begin{aligned}
& m \sqrt{\alpha-1}+\sqrt{\beta}=1 \\
& m^{2} \sqrt{\alpha-1}+\beta=1
\end{aligned}
$$


Lemma 2.3 (see [3, p. 230, Entry 5(ii)]). If $\beta$ has degree 3 over $\alpha$, then

$$
(\alpha \beta)^{1 / 4}+\{(1-\alpha)(1-\beta)\}^{1 / 4}=1 .
$$

Lemma 2.4 (see $[3$, p. 215, (24.22)]). If $\beta$ has degree 4 over $\alpha$, then

$$
\sqrt{\beta}=\left(\frac{1-(1-\alpha)^{1 / 4}}{1+(1-\alpha)^{1 / 4}}\right)^{2}
$$

Lemma 2.5 (see [3, p. 280-281, Entry 13(v) and (vi)]). If $\beta$ has degree 5 over $\alpha$, then

$$
\begin{aligned}
& m=\frac{1+\left((1-\beta)^{5} /(1-\alpha)\right)^{1 / 8}}{1+\left\{(1-\alpha)^{3}(1-\beta)\right\}^{1 / 8}} \\
& \frac{5}{m}=\frac{1-\left((1-\alpha)^{5} /(1-\beta)\right)^{1 / 8}}{1-\left\{(1-\alpha)(1-\beta)^{3}\right\}^{1 / 8}} .
\end{aligned}
$$

Lemma 2.6 (see [3, p. 314, Entry 19(i)]). If $\beta$ has degree 7 over $\alpha$, then

$$
(\alpha \beta)^{1 / 8}+\{(1-\alpha)(1-\beta)\}^{1 / 8}=1 .
$$

\section{Modular Identitites for $T(q)$}

In this section, we use Ramanujan's modular equations to prove certain modular identities for $T(q)$.

Theorem 3.1. One has

$$
T(q)=\frac{f(q)-f(-q)}{f(q)+f(-q)}
$$

Proof. Replacing $q$ by $q^{2}$ and the setting $a=q$ and $b=0$ in (1.7) and simplifying, we obtain

$$
\frac{\left(-q ; q^{2}\right)_{\infty}-\left(q ; q^{2}\right)_{\infty}}{\left(-q ; q^{2}\right)_{\infty}+\left(q ; q^{2}\right)_{\infty}}=\frac{q}{1-q^{2}}+\frac{q^{4}}{1-q^{6}}+\frac{q^{8}}{1-q^{10}}+\cdots
$$

Employing (1.6) and (1.9) in (3.2) and simplifying, we complete the proof.

Corollary 3.2. One has

$$
\frac{1+T(q)}{1-T(q)}=\frac{f(q)}{f(-q)}
$$


Proof. Dividing numerator and denominator on right-hand side of the identity in Theorem 3.1 by $f(-q)$ and simplifying, we complete the proof.

Theorem 3.3. One has

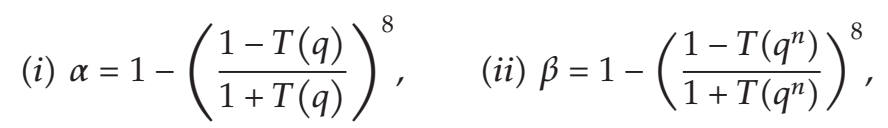

where $\beta$ has degree n over $\alpha$.

Proof. We employ Lemma 2.1 in Corollary 3.2 to complete the proof.

Theorem 3.4. Let $u=T(q)$ and $v=T(-q)$. Then,

$$
u+v=0
$$

Proof. Replacing $q$ by $-q$ in Corollary 3.2, we obtain

$$
\frac{1+T(-q)}{1-T(-q)}=\frac{f(-q)}{f(q)}
$$

Now, eliminating $f(q) / f(-q)$ between (3.6) and Corollary 3.2 and simplifying, we complete the proof.

Theorem 3.5. Let $u=T(q)$ and $v=T\left(q^{2}\right)$. Then,

$$
u^{2}-v-2 u^{2} v-u^{4} v+6 u^{2} v^{2}-v^{3}-2 u^{2} v^{3}-u^{4} v^{3}+u^{2} v^{4}=0
$$

Proof. Eliminating $m$ in (2.2) and then simplifying, we deduce that

$$
(1+\beta+(\beta-1) \sqrt{1-\alpha})^{2}-4 \beta=0
$$

From Theorem 3.3(i), we have

$$
\sqrt{1-\alpha}=\left(\frac{1-T(q)}{1+T(q)}\right)^{4}
$$

Now, employing Theorem 3.3(ii) with $n=2$ and (3.9) in (3.8) and factorizing using Mathematica, we obtain

$$
\begin{aligned}
(-1+v)^{8} & \left(u^{2}-v-2 u^{2} v-u^{4} v+6 u^{2} v^{2}-v^{3}-2 u^{2} v^{3}-u^{4} v^{3}+u^{2} v^{4}\right) \\
& \times\left(1+2 u^{2}+u^{4}-16 u^{2} v+6 v^{2}+12 u^{2} v^{2}+6 u^{4} v^{2}-16 u^{2} v^{3}+v^{4}+2 u^{2} v^{4}+u^{4} v^{4}\right)=0
\end{aligned}
$$


It can be seen that the first and the last factors in (3.10) do not vanish for $|q| \rightarrow 0$. So, by identity theorem, we have

$$
u^{2}-v-2 u^{2} v-u^{4} v+6 u^{2} v^{2}-v^{3}-2 u^{2} v^{3}-u^{4} v^{3}+u^{2} v^{4}=0
$$

Theorem 3.6. Let $u=T(q)$ and $v=T\left(q^{3}\right)$. Then,

$$
u^{3}-v-3 u^{2} v+3 u v^{2}+3 u^{3} v^{2}-3 u^{2} v^{3}-u^{4} v^{3}+u v^{4}=0
$$

Proof. From Lemma 2.3, we obtain

$$
\alpha \beta-\left(1-(1-\alpha)^{1 / 4}(1-\beta)^{1 / 4}\right)^{4}=0 .
$$

From Theorem 3.3, we deduce that

$$
\begin{gathered}
\alpha=1-\left(\frac{1-u}{1+u}\right)^{8}, \quad \beta=1-\left(\frac{1-v}{1+v}\right)^{8}, \\
(1-\alpha)^{1 / 4}=\left(\frac{1-u}{1+u}\right)^{2}, \quad(1-\beta)^{1 / 4}=\left(\frac{1-v}{1+v}\right)^{2},
\end{gathered}
$$

where $\beta$ has degree 3 over $\alpha$.

Employing (3.14) in (3.13) and factorizing using Mathematica, we arrive at

$$
\begin{aligned}
& \left(-u^{3}+v+3 u^{2} v-3 u v^{2}-3 u^{3} v^{2}+3 u^{2} v^{3}+u^{4} v^{3}-u v^{4}\right) \\
& \quad \times\left(-u+3 u^{2} v+u^{4} v-3 u v^{2}-3 u^{3} v^{2}+v^{3}+3 u^{2} v^{3}-u^{3} v^{4}\right)=0
\end{aligned}
$$

It can be seen that the second factor of (3.15) does not vanish for $|q| \rightarrow 0$, so by identity theorem, we have

$$
u^{3}-v-3 u^{2} v+3 u v^{2}+3 u^{3} v^{2}-3 u^{2} v^{3}-u^{4} v^{3}+u v^{4}=0
$$

Theorem 3.7. Let $u=T(q)$ and $v=T\left(q^{4}\right)$. Then,

$$
\begin{aligned}
u^{4}-v & -4 u^{2} v+2 u^{4} v-4 u^{6} v-u^{8} v+28 u^{4} v^{2}-7 v^{3}-28 u^{2} v^{3}+14 u^{4} v^{3}-28 u^{6} v^{3} \\
& -7 u^{8} v^{3}+70 u^{4} v^{4}-7 v^{5}-28 u^{2} v^{5}+14 u^{4} v^{5}-28 u^{6} v^{5}-7 u^{8} v^{5}+28 u^{4} v^{6}-v^{7}-4 u^{2} v^{7} \\
& +2 u^{4} v^{7}-4 u^{6} v^{7}-u^{8} v^{7}+u^{4} v^{8}=0
\end{aligned}
$$


Proof. Squaring the modular equation in Lemma 2.4 and simplifying, we obtain

$$
\beta-\left(\frac{1-(1-\alpha)^{1 / 4}}{1+(1-\alpha)^{1 / 4}}\right)^{4}=0 .
$$

From Theorem 3.3(i), we have

$$
(1-\alpha)^{1 / 4}=\left(\frac{1-T(q)}{1+T(q)}\right)^{2} .
$$

Now, employing Theorem 3.3(ii) with $n=4$ and (3.19) in (3.18) and simplifying, we complete the proof.

Theorem 3.8. Let $u=T(q)$ and $v=T\left(q^{5}\right)$. Then,

$$
u^{5}-v-5 u^{2} v+10 u^{3} v^{2}+5 u^{5} v^{2}-10 u^{2} v^{3}-10 u^{4} v^{3}+5 u v^{4}+10 u^{3} v^{4}-5 u^{4} v^{5}-u^{6} v^{5}+u v^{6}=0
$$

Proof. From Theorem 3.3, we obtain

$$
c:=(1-\alpha)^{1 / 8}=\left(\frac{1-u}{1+u}\right), \quad d:=(1-\beta)^{1 / 8}=\left(\frac{1-v}{1+v}\right)
$$

where $\beta$ has degree 5 over $\alpha$.

Employing (3.21) in (2.5), we find that

$$
\begin{aligned}
& m=\frac{c+d^{5}}{c\left(1+c^{3} d\right)} \\
& \frac{5}{m}=\frac{d-c^{5}}{d\left(1-c d^{3}\right)}
\end{aligned}
$$

respectively.

Eliminating $m$ between (3.22) and (3.23) and simplifying, we deduce that

$$
5 c d\left(1+c^{3} d\right)\left(1-c d^{3}\right)-\left(c+d^{5}\right)\left(d-c^{5}\right)=0 .
$$

Substituting for $c$ and $d$ from (3.21) in (3.24) and simplifying, we arrive at

$$
u^{5}-v-5 u^{2} v+10 u^{3} v^{2}+5 u^{5} v^{2}-10 u^{2} v^{3}-10 u^{4} v^{3}+5 u v^{4}+10 u^{3} v^{4}-5 u^{4} v^{5}-u^{6} v^{5}+u v^{6}=0
$$


Theorem 3.9. Let $u=T(q)$ and $v=T\left(q^{7}\right)$. Then,

$$
\begin{aligned}
& u^{8}-u v-7 u^{3} v-7 u^{5} v+7 u^{7} v+28 u^{6} v^{2}-7 u v^{3}-49 u^{3} v^{3}+7 u^{5} v^{3}-7 u^{7} v^{3}+70 u^{4} v^{4} \\
& \quad-7 u v^{5}+7 u^{3} v^{5}-49 u^{5} v^{5}-7 u^{7} v^{5}+28 u^{2} v^{6}+7 u v^{7}-7 u^{3} v^{7}-7 u^{5} v^{7}-u^{7} v^{7}+v^{8}=0
\end{aligned}
$$

Proof. From Lemma 2.6, we obtain

$$
\alpha \beta-\left(1-(1-\alpha)^{1 / 4}(1-\beta)^{1 / 4}\right)^{8}=0 .
$$

Again, from Theorem 3.3, we deduce that

$$
\begin{gathered}
\alpha=1-\left(\frac{1-u}{1+u}\right)^{8}, \quad \beta=1-\left(\frac{1-v}{1+v}\right)^{8}, \\
(1-\alpha)^{1 / 8}=\left(\frac{1-u}{1+u}\right), \quad(1-\beta)^{1 / 8}=\left(\frac{1-v}{1+v}\right),
\end{gathered}
$$

where $\beta$ has degree 7 over $\alpha$.

Employing (3.28) in (3.27) and simplifying using Mathematica, we arrive at

$$
\begin{aligned}
& u^{8}-u v-7 u^{3} v-7 u^{5} v+7 u^{7} v+28 u^{6} v^{2}-7 u v^{3}-49 u^{3} v^{3}+7 u^{5} v^{3}-7 u^{7} v^{3}+70 u^{4} v^{4} \\
& -7 u v^{5}+7 u^{3} v^{5}-49 u^{5} v^{5}-7 u^{7} v^{5}+28 u^{2} v^{6}+7 u v^{7}-7 u^{3} v^{7}-7 u^{5} v^{7}-u^{7} v^{7}+v^{8}=0 .
\end{aligned}
$$

\section{Explicit Evaluations of $T(q)$}

In this section, we establish some general theorems for the explicit evaluations of the continued fraction $T(q)$ and give examples.

For $q:=e^{-\pi \sqrt{n}}$, Ramanujan's two class invariants $G_{n}$ and $g_{n}$ are defined by

$$
G_{n}=2^{-1 / 4} q^{-1 / 24} x(q), \quad g_{n}=2^{-1 / 4} q^{-1 / 24} x(-q)
$$

The class invariants $G_{n}$ and $g_{n}$ are connected by the relation [4, p. 187, Entry 2.1]:

$$
g_{4 n}=2^{1 / 4} g_{n} G_{n}
$$

The singular modulus $\alpha_{n}$ is defined by $\alpha_{n}:=\alpha\left(e^{-\pi \sqrt{n}}\right)$, where $n$ is a positive integer and unique positive number between 0 and 1 satisfying

$$
\sqrt{n}=\frac{{ }_{2} F_{1}\left(1 / 2,1 / 2 ; 1 ; 1-\alpha_{n}\right)}{{ }_{2} F_{1}\left(1 / 2,1 / 2 ; 1 ; \alpha_{n}\right)} .
$$



(1.6)]:

Class invariants and singular moduli are intimately related by the equalities [4, p. 185,

$$
G_{n}=\left(4 \alpha_{n}\left(1-\alpha_{n}\right)\right)^{-1 / 24}, \quad g_{n}=\left(4 \alpha_{n}\left(1-\alpha_{n}\right)^{-2}\right)^{-1 / 24}
$$

An account of Ramanujan's class invariants and singular moduli can be found in Chapter 34 of Berndt's book [4].

Theorem 4.1. One has

$$
T\left(e^{-\pi \sqrt{n}}\right)=\frac{1-\left(1-\alpha_{n}\right)^{1 / 8}}{1+\left(1-\alpha_{n}\right)^{1 / 8}}
$$

Proof. We set $q:=e^{-\pi \sqrt{n}}$ in Theorem 3.3(i) and use the definition of singular moduli $\alpha_{n}$ and simplifying, we complete the proof.

In the scattered places of his first notebook [1], Ramanujan calculated over 30 singular moduli $\alpha_{n}$. See Chapter 34 of Berndt's book [4] for details. Thus, one can use Theorem 4.1 to find the values of $T\left(e^{-\pi \sqrt{n}}\right)$ if the corresponding values of $\alpha_{n}$ are known. For example, from [4, p. 281, Theorem 9.2], we note that

$$
\alpha_{2}=(\sqrt{2}-1)^{2}
$$

Employing (4.6) in Theorem 4.1, we calculate

$$
T\left(e^{-\pi \sqrt{2}}\right)=\frac{1-(-2+2 \sqrt{2})^{1 / 8}}{1+(-2+2 \sqrt{2})^{1 / 8}} .
$$

Many other values of $T\left(e^{-\pi \sqrt{n}}\right)$ can be computed by using the known values of $\alpha_{n}$.

Theorem 4.2. One has

$$
T\left(e^{-\pi \sqrt{n}}\right)=\left(\frac{g_{4 n}-2^{1 / 4} g_{n}^{2}}{g_{4 n}-2^{1 / 4} g_{n}^{2}}\right) .
$$

Proof. Dividing numerator and denominator of right-hand side of Theorem 3.1 and employing (1.6), we obtain

$$
T(q)=\frac{x(q)-x(-q)}{x(q)+x(-q)}
$$


Setting $q:=e^{-\pi \sqrt{n}}$, employing the definitions of $G_{n}$ and $g_{n}$ from (4.1) in (4.9) and simplifying, we obtain

$$
T\left(e^{-\pi \sqrt{n}}\right)=\frac{G_{n}-g_{n}}{G_{n}+g_{n}}
$$

Substituting for $G_{n}$ from (4.2) in (4.10) and simplifying, we complete the proof.

Theorem 4.2 implies that if we know the values of $g_{n}$ and $g_{4 n}$ for any positive number $n$, then corresponding values of $T\left(e^{-\pi \sqrt{n}}\right)$ can easily be calculated. Saikia [9] evaluated several values of $g_{n}$ and $g_{4 n}$ for positive number $n$. For example, noting from [9, Theorem 3.5], we have

$$
g_{3}=2^{-1 / 6}(2+\sqrt{3})^{1 / 8}, \quad g_{12}=2^{1 / 6}(2+\sqrt{3})^{1 / 8}
$$

Employing (4.11) in Theorem 4.2, we obtain

$$
T\left(e^{-\pi \sqrt{3}}\right)=\frac{2-2^{3 / 4}(2+\sqrt{3})^{1 / 8}}{2+2^{3 / 4}(2+\sqrt{3})^{1 / 8}} .
$$

Many other values of $T\left(e^{-\pi \sqrt{n}}\right)$ can be determined by using the values of $g_{n}$ and $g_{4 n}$ evaluated in [9].

\section{References}

[1] S. Ramanujan, Notebooks, vol. 1,2, Tata Institute of Fundamental Research, Bombay, India, 1957.

[2] S. Ramanujan, The Lost Notebook and Other Unpublished Papers, Narosa, New Delhi, India, 1988.

[3] B. C. Berndt, Ramanujan's Notebooks, part 3, Springer, New York, NY, USA, 1991.

[4] B. C. Berndt, Ramanujan's Notebooks, Part 4, Springer, New York, NY, USA, 1998.

[5] B. C. Berndt, "Flowers which we cannot yet see growing in Ramanujan's garden of hypergeometric series, elliptic functions, and $q^{\prime} s, "$ in Special Functions 2000: Current Perspective and Future Directions, J. Bustoz, M. E. H. Ismail, and S. K. Suslov, Eds., vol. 30, pp. 61-85, Kluwer Academic, Dordrecht, The Netherlands, 2001.

[6] B. C. Berndt, S. S. Huang, J. Sohn, and S. H. Son, "Some theorems on the Rogers-Ramanujan continued fraction in Ramanujan's lost notebook," Transactions of the American Mathematical Society, vol. 352, no. 5, pp. 2157-2177, 2000.

[7] S.-Y. Kang, "Some theorems on the Rogers-Ramanujan continued fraction and associated theta function identities in Ramanujan's lost notebook," The Ramanujan Journal, vol. 3, no. 1, pp. 91-111, 1999.

[8] S.-Y. Kang, "Ramanujan's formulas for the explicit evaluation of the Rogers-Ramanujan continued fraction and theta-functions," Acta Arithmetica, vol. 90, no. 1, pp. 49-68, 1999.

[9] N. Saikia, "Ramanujan's modular equations and Weber-Ramanujan's class invariants $G_{n}$ and $g_{n}$," Bulletin of Mathematical Sciences, vol. 2, no. 1, pp. 205-223, 2012. 


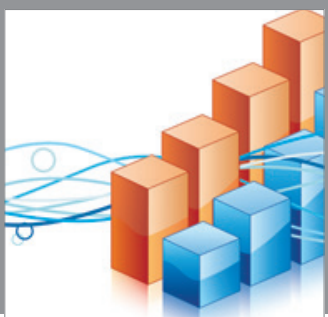

Advances in

Operations Research

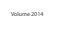

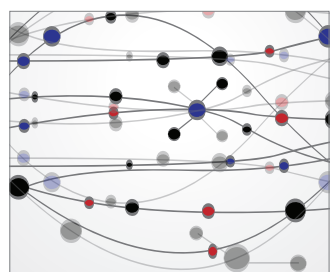

\section{The Scientific} World Journal
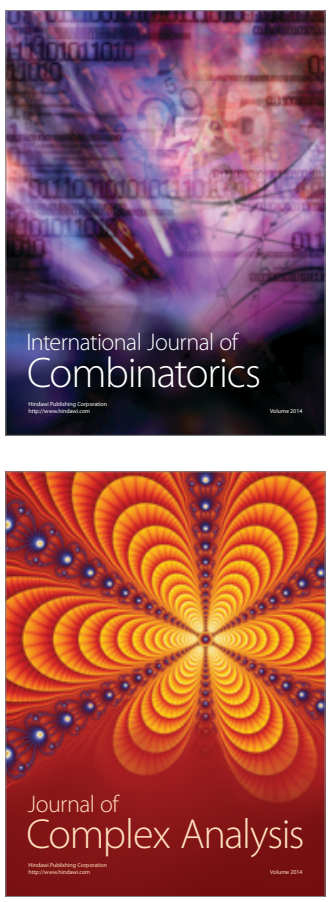

International Journal of

Mathematics and

Mathematical

Sciences
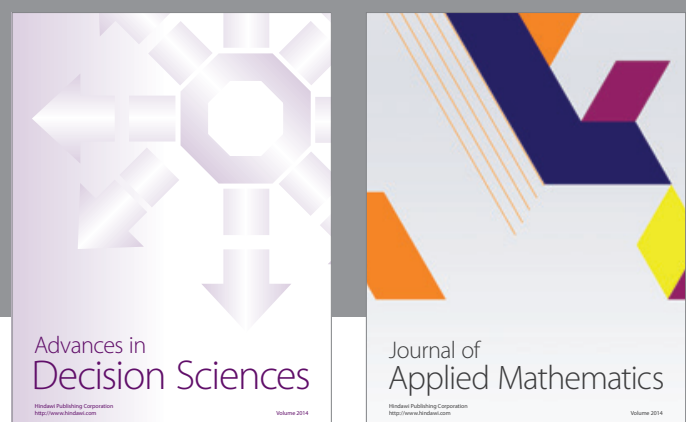

Journal of

Applied Mathematics
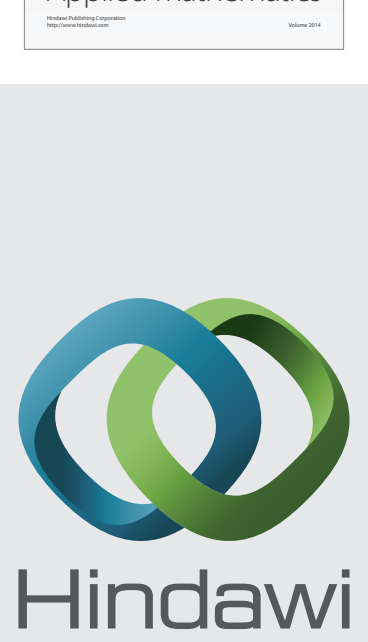

Submit your manuscripts at http://www.hindawi.com
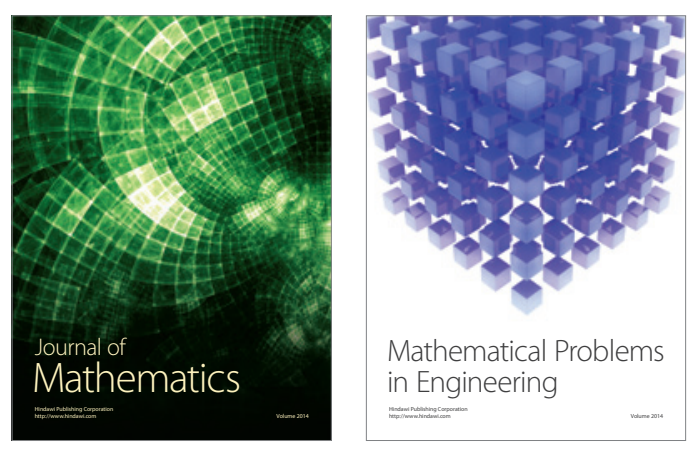

Mathematical Problems in Engineering
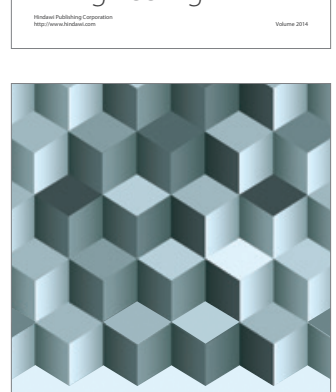

Journal of

Function Spaces
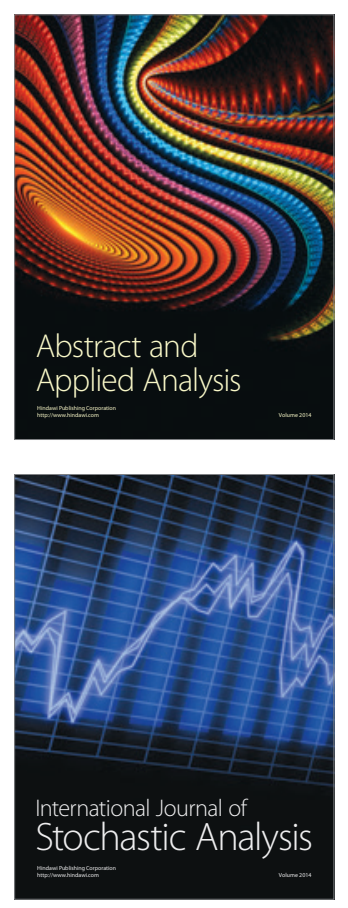

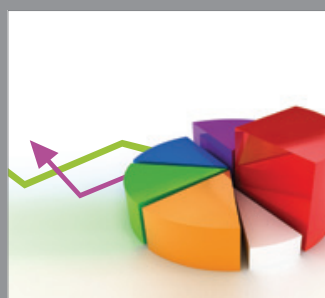

ournal of

Probability and Statistics

Promensencen
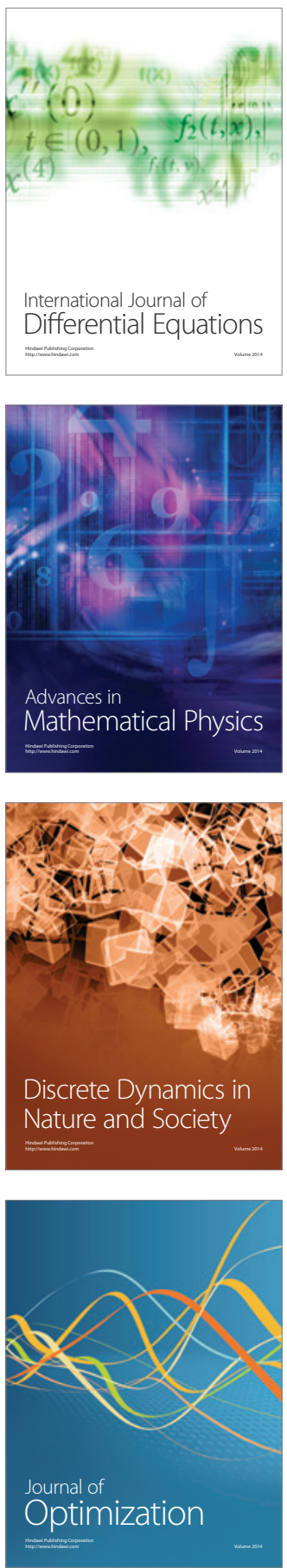